\title{
The Role of Mst1 in Lymphocyte Homeostasis and Function
}

\begin{abstract}
Jiali Cheng ${ }^{1}$, Yukai Jing ${ }^{1}$, Danqing Kang ${ }^{1}$, Lu Yang ${ }^{1}$, Jingwen Li', Ze Yu', Zican Peng ${ }^{1}$, Xingbo Li', Yin Wei ${ }^{2}$, Quan Gong ${ }^{3,4}$, Richard J. Miron ${ }^{5}$, Yufeng Zhang ${ }^{5}$ and Chaohong Liu' ${ }^{1 *}$

${ }^{1}$ Department of Microbiology, School of Basic Medicine, Tongji Medical College, Huazhong University of Science and Technology, Wuhan, China, ${ }^{2}$ Wuhan Children's Hospital, Tongji Medical College, Huazhong University of Science and Technology, Wuhan, China, ${ }^{3}$ Department of Immunology, School of Medicine, Yangtze University, Jingzhou, China, ${ }^{4}$ Clinical Molecular Immunology Center, School of Medicine, Yangtze University, Jingzhou, China, ${ }^{5}$ State Key Laboratory Breeding Base of Basic Science of Stomatology (Hubei-MOST) and Key Laboratory of Oral Biomedicine Ministry of Education, School and Hospital of Stomatology, Wuhan University, Wuhan, China
\end{abstract}

The Hippo pathway is an evolutionarily conserved pathway crucial for regulating tissue size and for limiting cancer development. However, recent work has also uncovered key roles for the mammalian Hippo kinases, Mst1/2, in driving appropriate immune responses by directing $T$ cell migration, morphology, survival, differentiation, and activation. In this review, we discuss the classical signaling pathways orchestrated by the Hippo signaling pathway, and describe how Mst1/2 direct T cell function by mechanisms not seeming to involve the classical Hippo pathway. We also discuss why Mst1/2 might have different functions within organ systems and the immune system. Overall, understanding how Mst1/2 transmit signals to discrete biological processes in different cell types might allow for the development of better drug therapies for the treatments of cancers and immune-related diseases.

Keywords: hippo, lymphocyte, migration, proliferation, apoptosis

\section{INTRODUCTION}

The evolutionarily conserved Hippo pathway was first identified in Drosophila and comprises several kinases, adaptor proteins, and transcription factors. Mammalian orthologs for each of the core Drosophila Hippo proteins are summarized in Figure 1 and include Mst1/2 (ortholog of Hpo), Salv1 (ortholog of Sav), Lats1/2 (ortholog of Wrts), Mob1A/B (ortholog of Mats), YAP/TAZ (orthologs of Yki), and TEAD1-4 (orthologs of Sd) (1-6). Mst1/2 is a member of the STE20 protein kinase family and contains an $\mathrm{N}$-terminal kinase domain and a C-terminal SARAH domain that allows Mst1/2 to form homo or heterodimers (7). The SARAH domain of Mst1/2 also binds to the scaffolding protein, Salv1, resulting in the phosphorylation of Salv1 and subsequent increase of Mst1/2 activity $(8,9)$. Mob1A/B is also a substrate of Mst1/2, which binds and promotes the activation of the kinases, Lats1/2 (10). The Lats1/2 PPxY domain binds the WW domain of YAP/TAZ, which allows Lats $1 / 2$ to recognize the HXRXXS sequence of YAZ/TAZ and phosphorylate all five serine sites (11-13). This phosphorylation event sequesters YAP/TAZ in the cytoplasm by allowing the 14-3-3 family of regulatory proteins to bind. Subsequently, YAP/TAZ are unable to cooperate with the TEAD transcription factors that promote oncogene transcription and cellular proliferation and are also subjected to degradation (14-17). Therefore, the activation of Hippo induces cell apoptosis by downregulating cell proliferation signaling. Indeed, inactivation of canonical Hippo signaling induces cancer, which is supported by many clinical case reports (18-20). 


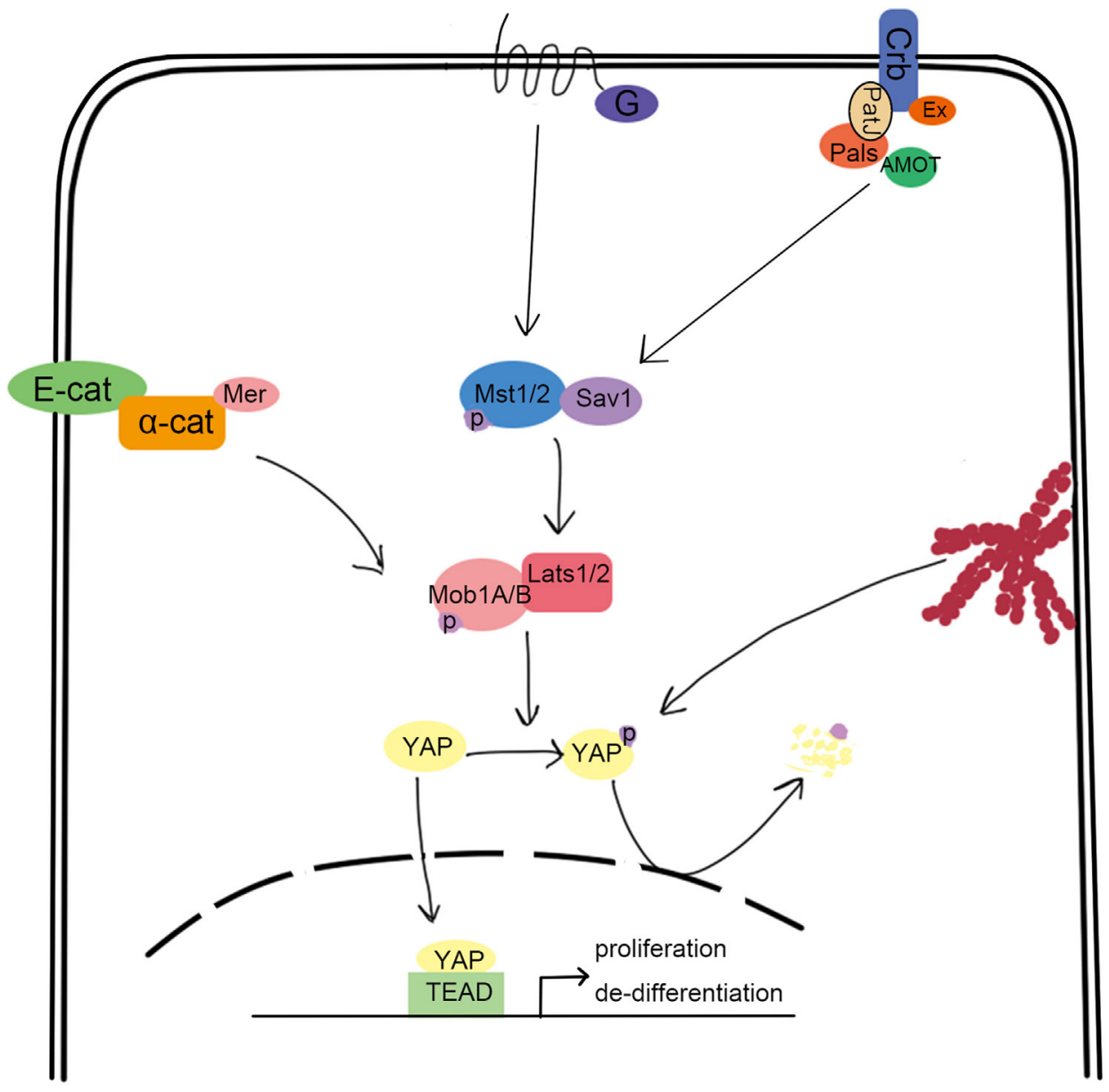

FIGURE 1 | Overview of the canonical hippo signaling pathway. Signals such as mechanical stress, and GPCR can activate Mst1/2, bound with Sav1 through the SARAH domain. The activated Mst1/2 kinases phosphorylate and activate Lats 1/2, which can interact with YAP through their respective PPXY and WW domains. Lats 1/2 then phosphorylate YAP, which traps it in the cytoplasm upon binding to 14-3-3. This results in the loss of YAP function, which is involved in promoting the transcription of cell proliferation-related genes.

Apart from cancer and tissue development, Hippo signaling proteins also play an important role in the immune system, including $\mathrm{T}$ cell development, survival, trafficking, and activation. Moreover, several recent studies have demonstrated that Mst1/2 regulates $\mathrm{T}$ cell biology independently of the canonical Hippo signaling pathway. In the following sections of this article, the relationship between Hippo/Mst1/2 and lymphocyte homeostasis, adhesion, proliferation, apoptosis, and differentiation is discussed. We also discuss why the Hippo signaling may differ in organ systems and the immune system.

\section{T CELL DEVELOPMENT AND PERIPHERAL HOMEOSTASIS OF PERIPHERAL T CELL ARE AFFECTED BY Mst1}

T lymphocytes originate from hematopoietic stem cells found in the bone marrow and complete development in the thymus, ultimately developing into mature, $\mathrm{CD} 4^{+}$, or $\mathrm{CD}^{+}$SP lymphocytes whose TCRs are restricted by self-MHC molecules and are not auto-reactive. The maturation of T cells in the thymus largely depends on their chemokine-mediated migration from the thymic cortex to the medulla (21). During their migration from the superficial cortex to the inner cortex, $\mathrm{CD} 4^{-} \mathrm{CD} 8^{-} \mathrm{DN}$ thymocytes undergo $\alpha \beta T C R$ gene rearrangement to develop into $\mathrm{CD}^{+} \mathrm{CD}^{+} \mathrm{DP}$ cells. Then, DP thymocytes become $\mathrm{CD} 8^{+}$ or $\mathrm{CD}^{+}{ }^{+} \mathrm{CD} 69^{\text {hi }} \mathrm{SP}$ cells in response to their respective $\mathrm{MHC}$ I or MHC II-restricted cues presented by cTECs present in the cortex, which is called positive selection (22). High-avidity self-antigen (presented by Aire ${ }^{+}$ICAM-1 hi mTECs in the medulla)-TCR interactions trigger apoptosis in SP thymocytes, a process called negative selection that induces central immune tolerance. Similarly, the development of Treg in the thymus is partly dependent upon a strong self-ligand signaling (23). Mst1/2 regulate key steps of thymocyte development. The population and distribution of DP thymocytes in $M s t 1 / 2^{-/-}$mice is unchanged (24), likely because Mst1/2 are expressed at low levels in DP thymocytes (25). However, CD69 ${ }^{\text {hi }} \mathrm{CD}^{+}$and $\mathrm{CD}^{+}$SP thymocytes are severely reduced in $M s t 1^{-/-}$mice (26), suggesting that Mst1 
deficiency impairs the positive selection at the transition from the DP to SP stage. While Dong et al. did not find such a reduction of $\mathrm{CD}_{6} 9^{\text {hi }} \mathrm{CD}^{+}$and $\mathrm{CD} 8^{+} \mathrm{SP}$ thymocytes in $M s t 1 / 2^{-/-}$mice (24). The population of $\mathrm{Mst}^{-/-} \mathrm{HSA}^{\mathrm{lo}} \mathrm{V} \beta 5^{+} \mathrm{CD}^{+} \mathrm{SP}$ thymocytes is significantly increased and the number of $\mathrm{Mst}^{-/-} \mathrm{FoxP}^{+} \mathrm{CD}^{+}$ Treg in the thymus is decreased, suggesting an additional role for Mst1 in negative selection and Treg development in the thymus.

The interaction between SP thymocytes and mTECs is mediated by LFA-1, which binds ICAM-1 on the mTECs to influence negative selection. In vitro, the migration velocity of $M s t 1^{-/-}$ immature SP $\mathrm{CD}^{+} \mathrm{T}$ cells on the ICAM-loaded membrane decreases dramatically when stimulated by CCL21. Moreover, the conjugation between $\mathrm{Mst}^{-/-}$immature $\mathrm{CD} 4^{+} \mathrm{SP}$ and cognate Aire ${ }^{+}$ICAM- ${ }^{+}$mTEC is unstable, due to defective ICAM-1/LFA-1 clustering (26). These findings collectively provide evidence that Mst1 plays an important role in LFA-1/ICAM-1-related thymocyte migration and antigen recognition, therefore regulating the $\mathrm{T}$ cell development in thymus. How Mst1 regulates LFA function is discussed in a later section.

Mature T lymphocytes continuously migrate from the thymus toward the peripheral lymphoid system. In $M s t 1^{-/-}$mice, the number of peripheral $\mathrm{T}$ cells is dramatically decreased with an unbalanced distribution of different subsets, including reduced frequency of naïve $\mathrm{T}$ cells and increased frequency of $\mathrm{CD}^{+} /$ $\mathrm{CD}^{+}$effector/memory $\mathrm{T}$ cells (27). Several factors account for the defects in peripheral $\mathrm{T}$ cell homeostasis in $\mathrm{Mst1}^{-/-}$mice. First, histological analysis of the thymus showed that mature $M s t 1^{-1-}$ SP thymocytes accumulate in the perivascular space around the thymus, consistent with inefficient egress of mature lymphocytes (28). Second, $M s t 1^{-1-}$ naïve $\mathrm{T}$ cells have a higher level of proliferation and apoptosis in response to TCR signaling. $M s t 1^{-1-}$ naïve $\mathrm{T}$ cells presumably are activated with a lower threshold stimulation in vivo and are deleted thereafter through apoptosis, consistent with reduced frequency of naïve T cells (29). It is also proposed that the high levels of proliferation are due to the dysfunction of $\mathrm{Mst}^{-1-}$ Treg cells (discussed more in a later section) (27, 30, 31). How Mst1 regulates the egress of lymphocyte and the apoptosis, proliferation will be illustrated in detail in a later section of this review.

In summary, Mst1 deficiency results in inefficient integrindependent thymocyte migration and antigen recognition, leading to failed positive and negative selection and decreased numbers and altered activation of peripheral lymphocytes. Thus, Mst1 has a profound role in shaping immunological responses by influencing thymocyte development and lymphocyte activation.

\section{Mst1 ASSOCIATED WITH LFA-1 CONTROLS THE MIGRATION OF LYMPHOCYTE}

LFA-1-mediated adhesion to epithelial cells or APC is important for lymphocyte homing and interstitial trafficking. LFA-1 also contributes to the formation of stable immunological synapses in which TCRs bind their specific pMHC, leading to the appropriate activation of T cells. LFA-1 is constitutionally expressed in lymphocytes in a low affinity form. To fulfill its function in directing cell migration and adhesion, LFA-1 undergoes affinity maturation and clustering. The key point in this enhanced affinity is the separation of the $\alpha \mathrm{L}$ and $\beta 2$ domains, which elongates the endoplasmic segments of LFA-1 (32). Mst1 has been demonstrated to be key for LFA-1 functions in T cells. In vitro, $\mathrm{Mst}^{-1-} \mathrm{T}$ cells are less motile on ICAM-1 when exposed to CCL21 or soluble anti-CD3 antibody. Further, LFA-1 does not undergo affinity maturation in $M s t 1^{-1-} \mathrm{T}$ cells (33). Mechanistically, phosphorylated ITAMs on the TCR recruit and lead to the phosphorylation of ZAP-70. In turn, activated ZAP-70 phosphorylates SLP-76, allowing the SH2 domain of SLP-76 binding to bind ADAP at YDDV sites. The SH3 domain of SKAP1/SKAP55 then binds to the proline-rich region of $\operatorname{ADAP}(34,35)$. This ADAP-SKAP1 interaction inhibits degradation of SKAP1 and allows antigen signals to be transmitted to promote affinity maturation of LFA-1 $(36,37)$. This pathway is summarized in Figure 2. More recently, ADAP-SKAP/SKAP55 were found to interact with Mst1 in two independent complexes: one that contains RAPL and Rap1; the other that contains RIAM, Rap1, and TALIN or kindlin-3 (38). RAPL is a Rap1 effector protein that directs RAPL/Mst1/Rap1 to the cytomembrane (39). Similarly, RIAM directs the relocalization of RIAM/Mst1/TALIN complexes (40). Then, the FERM domain of TALIN binds to LFA$1-\beta 2$ domain, inducing LFA- 1 activation. Mst1 deficiency affects the relocation of RAPL/Rap1 and RIAM/Rap1 to the cytomembrane, as well as the activation of LFA-1. On the other hand, the deficiency in RAPL or Rap1 inhibits the activation of Mst1, leading to failed LFA-1-dependent lymphocyte migration (33), which suggests that the structure and kinase activity of Mst1 are both indispensable for LFA-1 activation. How is Mst1 activated in this pathway? It is reported that Rap1 is activated by TCR or chemokine signals, and then recruits SARAH domain-mediatedhetero-dimerization Mst1/RAPL controls Mst1 localization and activation at the membrane, facilitating the activation of LFA-1. Of note, it is reported that kindlin-3 regulated by active Mst1 via NDR1/2 interacts with cytoskeleton at the inner pSMAC to regulate the formation and stability of immunological synapse (41). However, the underlying mechanism needs more explanation. There are some arguments that Mst1 competes with RAPL for binding sites on SARAH domain of SKAP1/SKAP55, which remains to be illustrated.

LFA redistribution, another key factor in LFA-1-dependent migration, is associated with vesicle transportation, in which RapL/Rap1/Mst1 complex plays an important role (Figure 2). After chemokine stimulation in $M s t 1^{-1-}$ or $R A P L^{-l-} \mathrm{T}$ cells, the distribution of LFA-1 is dispersed rather than clustered $(42,43)$. Rab13, a member of the small Ras-related GTP binding protein family, is a key effector of Mst1 in the process of LFA-1 redistribution $(44,45)$. Immunoprecipitation experiments revealed that Mst1 preferentially interacts with active Rab13. Furthermore, Rab13 has little influence on Mst1 phosphorylation levels, but Mst1 deletion results in a large decrease in Rab13 activity (45). Rab13 is an important effector of Mst1-dependent LFA-1 function, since LFA-1 is unable to cluster at the leading edge or the immune synapse when stimulated by chemokines and antigen in $\mathrm{Rab} 13^{-1-}$ T cells (45). Mechanically, when stimulated with cytokines, Rab13 can be switched into an active, GTP-bound state through Mst1activated DENNC1C, a GEF for Rab13 $(45,46)$. In turn, activated 


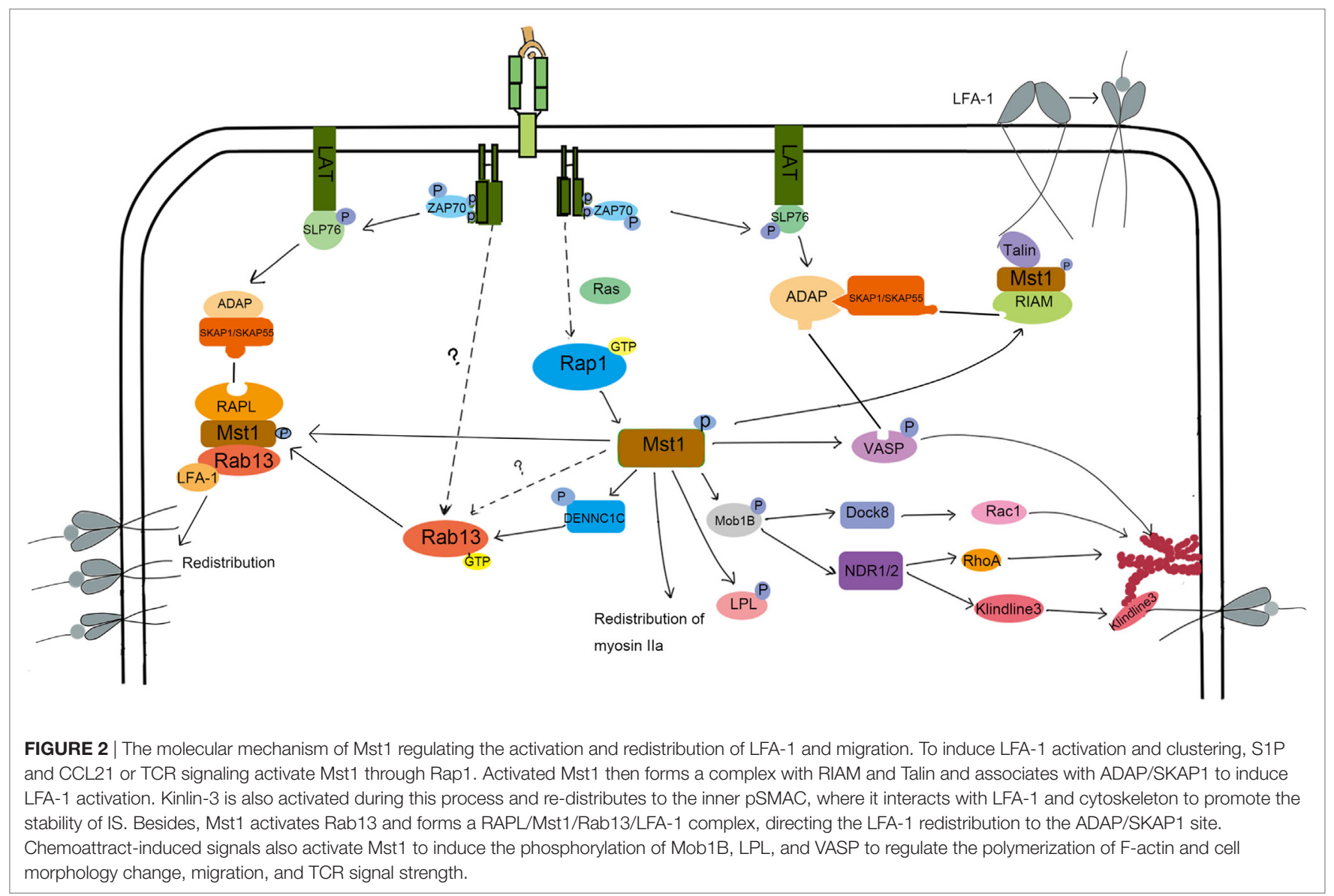

Rab13 associates with the LFA and Mst1/RAPL complex, which localizes LFA-1 to the destined leading edge. Indeed, active Rab13 co-localizes with Mst1 in the plasma membrane and peri-nuclear regions, but did not translocate to the leading edge together with LFA-1 until after CXCL12 stimulation. In contrast, LFA-1 does not cluster at the leading edge or promote adhesion and migration of T cells after CXCL12 stimulation when Rab13 is inactive. These results suggest that Rab13 activity is necessary but not sufficient for CXCL12-induced redistribution of LFA-1.

What other Mst1 effectors might promote LFA-1 clustering upon CXCL12 stimulation? Mst1 can also phosphorylate VASP at the serine 157, which allows ADAP to bind to VASP (45). VASP is known to facilitate F-actin polymerization and induce the extension of actin filaments (47). Therefore, Mst1 influences cell shape by controlling F-actin reorganization in a VASP-dependent manner. In doing so, VASP-mediated actin reorganization cooperates with Mst1/Rab13/RAPL to transport the active LFA-1 to the adhesive site, regulating the adhesion and movement of lymphocytes. Mst1 also organizes the immunological synapse by the regulating vesicle transport of the TCR to the cSMAC. Indeed, VSP4, a biomarker of TCR-enriched vesicle (48), and Rab8 or Rab11, proteins that mediate TCR vesicle transport $(41,49)$, are not enriched at the immunological synapse in Mst1/2-deficient T cells.

In conclusion, Mst1 regulates the activation and redistribution of LFA-1 to affect T cell migration and TCR signaling. The ADAP/
SKAP1 complex is an important adaptor that regulates the accurate relocation of RAPL/RAP1/Mst1/Rab13 and RAPL/RAP1/ Mst1/TALIN or kindlin-3 complexes to the adhesive site. Rap1/ RAPL may be the activator of Mst1 downstream of chemokines like CXCL12, whose physical structure contributes to the stability of the above complex. Further, Mst1 kinase activity facilitates the polymerization of F-actin and vesical transportation of LFA-1 through VASP and Rab13.

\section{THE ROLE OF MSt1 IN THE REGULATION OF CYTOSKELETON REARRANGEMENT IN T CELLS}

Cell motility is dependent upon the depolymerization and polymerization of actin filaments, which facilitates the cell's morphology, protrusion, and uropod formation. When adhesion molecules cluster at the leading edge and detach at the trailing edge, the cell body is pulled in the direction of chemokine gradients that induce migration (50). The protein LPL is abundant in lymphocytes, especially in the actin structures called lamellipodium. Mst1 directly phosphorylates LPL at threonine 89, inducing the formation of lamellipodium and promoting cell migration (51). Additionally, Mst1 restricts the distribution of myosin IIa, which co-localizes with F-actin, to affect the cell morphology and promote the localization of LFA-1 to the uropod and trailing edge 
(52). Polymerization of F-actin is also regulated by localized activation of the Rho family GTPase (53). Experiments have shown that antigen or chemokine-induced actin polymerization and Rho activation are blocked in $M s t 1^{-1-} \mathrm{CD} 4^{+} \mathrm{T}$ cells. The ability of Mst1 to influence Mob1/Dock8 or Mob1/NDR1/2 activation may participate in controlling Rho activation and actin polymerization as summarized in Figure 2 and discussed below $(25,28)$.

Chemokines like S1P or CCL21 induce the phosphorylation of Mob1B in an Mst1-dependent manner, and phosphorylated Mob1B interacts with and activates Dock8 (25). Dock8 is a GEF that contains an $\mathrm{N}$-terminal domain and a C-terminal domain, which localize Dock8 to the membrane and regulate its GEF activity, respectively (54). Dock8 then promotes Rac1 activation and subsequent cell migration. Activated Mob1B can also interact with NDR1/2, which allows for the Mst1-dependent phosphorylation of NDR1/2 and induction of Rho GTPase activation. This axis was found to be crucial for facilitating thymocyte egress, homing, and migration (28). It is interesting that the S1P or CCL21-induced phosphorylation of Mob1B is decreased dramatically in $M s t 1^{-1-} \mathrm{T}$ cells but there is no effect on Lats1/2 and NDR1/2 phosphorylation, which indicates that other regulators independent of Mst1 phosphorylate DNR1/2 and activate Rho GTPase (25). These results suggest that Mob1B is a pivotal substrate of Mst1 in chemokine-induced migration. It should be noted that different chemokines seem to activate different small G proteins: CCL21, CCL17, CCL25, CCL19 induce Rac1 activation; S1P induces RhoA activation. As Mst1/2 deficiency leads to impaired Racl or RhoA activity, it appears Mst1 plays a role of "relay station" in these signaling pathways to influence cell migration following chemokine stimulation.

In conclusion, Mst1 directly interacts with LPL and restricts the spatial distribution of myosin IIa, regulating the polarization of T cells. Additionally, Mst1 can affect the cytoskeleton rearrangement of chemokine-stimulated $\mathrm{T}$ cells by regulating the activity of the Rho family GTPase, in which Mob1 and NDR1/2 phosphorylated by activated Mst1 help to fulfill the activation of Racl and RhoA.

\section{Mst1 AND LYMPHOCYTE PROLIFERATION AND APOPTOSIS}

As we noted earlier, $M s t 1^{-1-}$ mice have profound reductions in peripheral $\mathrm{T}$ cell numbers and alterations in the antigen-induced apoptosis and proliferation (55). However, there is some controversy as to the role of Mst1 in these processes. Some reports suggested that, upon anti-CD3 and anti-CD28 antibody stimulation, $M s t 1^{-/-}$naïve T cells exhibit a higher level of proliferation, while $M s t 1^{-1-}$ effector/memory T cells do not. However the production of cytokines is enhanced in both naïve and effector/memory $\mathrm{T}$ cell subsets when Mst1 is deleted (27). It was found that the expression of Mst1 is nearly 10-fold higher in naïve T cells than effector/memory $\mathrm{T}$ cells, suggesting that Mst1 levels dictate a threshold for antigen-induced proliferation of naïve $\mathrm{T}$ cells. Therefore, when the restriction is removed upon Mst1 deletion, naïve $\mathrm{T}$ cells undergo a rapid proliferation after stimulation with antigen. The next question is how does Mst1 deficiency accelerate the proliferation of naïve $\mathrm{T}$ cells after being activated? Thus
TABLE 1 | The events affected and unaffected in Mst1 ${ }^{-/-}$T cell proliferation.

\begin{tabular}{|c|c|c|c|}
\hline Events & Affected & Unaffected & Effect \\
\hline Over-expression of PD-1 & $\sqrt{ }$ & & $\begin{array}{l}\text { Higher level of activation- } \\
\text { induced Mst1 }{ }^{-/-} \mathrm{T} \text { cell } \\
\text { apoptosis }\end{array}$ \\
\hline $\begin{array}{l}\text { Reduced-expression of } \\
\text { FoxO1/3 }\end{array}$ & $\sqrt{ }$ & & $\begin{array}{l}\text { Higher level of Mst1 } 1^{-/} \\
\text {naïve T cell apoptosis }\end{array}$ \\
\hline JUK activation & $\sqrt{ }$ & & $\begin{array}{l}\text { Higher level of Mst1-/- } \\
\text { effector/memory T cell } \\
\text { apoptosis }\end{array}$ \\
\hline Erk activation & & $\sqrt{ }$ & / \\
\hline P38 activation & & $\sqrt{ }$ & / \\
\hline Akt activation & & $\sqrt{ }$ & / \\
\hline IkB activation & & $\sqrt{ }$ & / \\
\hline Lats $1 / 2$ activation & & $\sqrt{ }$ & / \\
\hline YAP activation & & $\sqrt{ }$ & / \\
\hline
\end{tabular}

far, no convincing evidence has been provide, but the canonical substrates of Mst1, Lats1/2, and YAP do not participate in this process as summarized in Table $\mathbf{1}(25,27)$.

In contrast to the above study, other groups have found that antigen-induced proliferation of $M s t 1^{-/}$naïve T cells is hindered, with cell cycle progression being partially blocked at the G1/S transition (56). Annexin-V staining in activated total peripheral $M s t 1^{-1-} \mathrm{T}$ cells is upregulated and the expression of $\mathrm{Bcl}-2$ is downregulated (27), which is consistent with increased cell apoptosis contributing to defects in activation-induced proliferation. An important note is that $M s t 1^{-1-} \mathrm{T}$ cells do not exhibit increased apoptosis before activation, suggesting that these defects are related to activation. Activated $M s t 1^{-1-} \mathrm{T}$ cells also have increased expression PD-1 (27), so peripheral CD4 ${ }^{+} \mathrm{T}$ cell apoptosis could be induced by PD-L1 present in different environments where $\mathrm{T}$ cells are inclined for apoptosis. The molecular mechanism of how Mst1 inhibits PD-1 expression on T cells remains to be elucidated. Canonical TCR signaling pathways inducing Lck, ZAP-70, PLC $\gamma 1$, and $\mathrm{Ca}^{2+}$ signaling are not affected in Mst1-deficient $\mathrm{T}$ cells. Further, Mst1-deficient T cells have normal activation of Erk, p38, Akt, and phosphorylation of IкB (indicator of NF- $\kappa \mathrm{B}$ ).

Apart from the high levels of $\mathrm{PD}-1, \mathrm{Mst1}^{-/-}$naive $\mathrm{T}$ cells have reduced levels of FoxO1/3 (55), transcription factors that facilitate oncogenic gene expression and inhibit cell apoptosis. It is reported that Mst1 increases the expression level of FoxO1/3 by phosphorylating its forkhead domain and therefore inducing its nuclear entrance (57). Another report found that in TCRstimulated $\mathrm{Mst}^{-1-}$ effector/memory T cells, the phosphorylation of Erk, p38, AKT, and IкB are also not altered. However, there is an increased activation of JNK, indicating that JNK hyperactivation might also promote apoptosis in effector/memory Mst $^{-1-} \mathrm{T}$ cells (57). It is a paradoxical that Mst1 deficiency leads to enhanced apoptosis in naïve and effector/memory T cells, while the canonical function of Hippo signaling is to inhibit proliferation and induce apoptosis. These differences may be accounted for by a non-canonical function of Mst1/2, as Lats1/2 and YAP functions are not known to be regulated by Mst1 in T cells. These findings indicate that Mst1 signaling pathway is special in lymphocytes and the mechanism it functions in the regulation of lymphocyte proliferation and apoptosis is not clearly illustrated. 
In conclusion, $\mathrm{Mst}^{-/-}$naïve $\mathrm{CD} 4^{+} \mathrm{T}$ cells are prone to apoptosis and express higher levels of PD-1 and less FoxO1/3 after stimulation, which results in the defect immune responses. Effector/ memory $\mathrm{Mst}^{-1-} \mathrm{CD} 4^{+} \mathrm{T}$ cells show a high level of activationinduced apoptosis, which may related with the increased activity of JNK.

\section{Mst1 AND T CELL DIFFERENTIATION}

Some researchers stressed that lymphocyte differentiation, but not proliferation and apoptosis, is regulated by the Hippo signaling pathway. The terminal differentiation of Treg and $\mathrm{CD}^{+} \mathrm{T}$ cells differentiation, but not proliferation are also dependent upon Mst1 (58). Additionally, the differentiation of naïve $\mathrm{CD}^{+} \mathrm{T}$ cells into Th17 and Th2 cells is also regulated by Mst1 Each of these points is discussion in more detail below and are summarized in Figure 3.

The differentiation and activation of Treg cells are restrained in $M s t 1^{-1-}$ mice, leading to a defect in immune tolerance that results in the over-activation and proliferation of naïve $\mathrm{T}$ cells. Indeed, in $\mathrm{Mst}^{-1-}$ mice, the number of FoxP3 ${ }^{+} \mathrm{CD} 4^{+}$Treg is markedly reduced. Similarly, the conversion of naïve $\mathrm{CD} 4^{+} \mathrm{T}$ cells into FoxP $3^{+} \mathrm{CD} 4^{+}$Treg in response to antigen and TGF- $\beta$ stimulation is also severely reduced in the absence of Mst1. Mechanistically, Mst1 induces FoxP3 expression, the transcription factor that defines the Treg lineage and function by facilitating the expression of CD25 and Nrp1 while suppressing the expression of IL-2 and IFN- $\gamma$ (59). Mst1 can regulate the expression of FoxP3 at the transcriptional and posttranslational levels. Tao's group suggests that Mst1 promotes the expression of FoxP3 by antagonizing the activity of AKT, which antagonizes the FoxO1/3a-dependent transcription of FoxP3 (31). The stability and function of
FoxP3 requires proper acetylation by lysine acetyl transferases. Therefore, deacetylases like Sirt1, which interacts with FoxP3 and deacetylates FoxP3, inhibit the function of FoxP3 and Treg differentiation (60). Mst1 can protect FoxP3 from being deacetylated by Sirt1, which subsequently enhances the transcriptional activity of FoxP3 to induce Nrp1 and CD25 expression and Treg cell differentiation and function (61). Mst1 exerts its control over Sirt1 by blocking its binding to FoxP3 and by acting in a kinasedependent manner to inhibit Sirt1 activation. Notably, Mst1 can induce FoxO1/3 nuclear translocation by phosphorylating its forkhead domain in granule neurons (62). FoxP3 is also a forkhead protein, thus suggesting another potential layer by which Mst1 could regulate FoxP3 expression and function.

Further research of $M s t 1^{-1-}$ Treg found that Mstl affects the function of Treg by regulating their trafficking and interactions with DC. In secondary lymphoid organ, Treg and naïve T cells move rapidly to recognize the antigen. After the recognition of antigen presented by APC, naïve T cells are arrested and firmly attached with APC, which promotes naïve T cell activation. Meanwhile, Treg move relatively slowly and only temporarily interact with DC, showing a "stop and go" model to exert an immunomodulation function. Adhesion molecule-mediated interactions between APC and Treg are fundamental for Treg functions. The trafficking velocity of $M s t 1^{-/-}$Treg is slower than WT Treg, indicating that Mst1-deficient Treg may encounter fewer APC at a slower rate. In vitro, conjugate formation between $M s t 1^{-1-}$ Treg and antigen-specific DC is reduced, which is associated with altered dispersions of LFA-1 and pMHC clusters in the membrane (30). Additionally, Mst1 ${ }^{-/-}$Treg express higher levels of CD86, suggesting that Mst1 regulates the function of Treg through LFA-1-mediated adhesion to DC and not through CD28 co-stimulatory mechanisms. Consistent with the dysfunction of

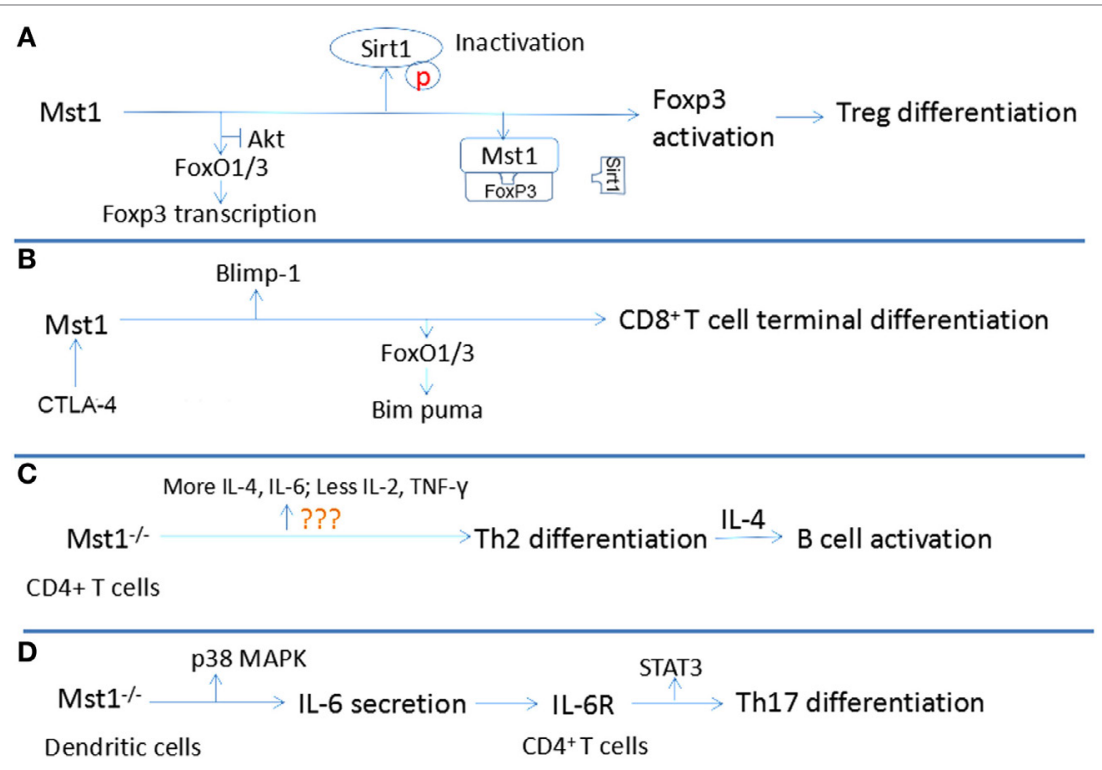

FIGURE 3 | Relationship between Mst1 and T cell differentiation. (A) Mst1 promotes the activation of Foxp3 through antagonizing the activity of AKT and Sirt1, inducing the differentiation of regulatory T cell (Treg). (B) Combination of CD80 to CTLA-4 activates Mst1, which regulates the activity of Blimp1 and Bim puma, facilitating the terminal differentiation of $\mathrm{CD} 8^{+} \mathrm{T}$ cells. (C) Mst1 $1^{-/-} \mathrm{CD} 4^{+} \mathrm{T}$ cells are inclined to differentiate to $\mathrm{T}$ helper cells (Th)2, which induce B cell activation by producing interleukin (IL)-4. (D) Mst1 ${ }^{-/-}$dendritic cells secret more IL-6, which regulates Th17 differentiation by agonizing IL-6R of CD4+ T cells. 
$M s t 1^{-/-}$Treg, $M s t 1^{-/-}$Treg cannot suppress colitis induced by the adoptive transfer of CD62 $\mathrm{L}^{\text {hi }}$ naïve $\mathrm{T}$ cells into healthy mice. In summary, Mst1 deficiency leads to the impaired differentiation and function of Treg, which may contribute to the over-activation and proliferation of naive $\mathrm{CD} 4^{+} \mathrm{T}$ cells. The underlying mechanism is that Mst1 regulates the immunological synapse formation between Treg and DC. Whether a difference exists in the module of Treg and DC interaction remains to be explored.

Mst1 can also control the terminal differentiation of $\mathrm{CD}^{+}$ $\mathrm{T}$ cells. It is known that $\mathrm{CD} 8^{+} \mathrm{T}$ cells will develop into effector $\mathrm{CD}^{+} \mathrm{T}$ cells that attack invading pathogens, and memory $\mathrm{CD}^{+} \mathrm{T}$ cells that are prepared for a potential future invasion by a previously encountered pathogen. An important point is that $\mathrm{CD}^{+} \mathrm{T}$ cells activated by antigen and cytokines should differentiate within a proper time interval after expansion to ensure an effective immune response. If too early, there are not enough effector $\mathrm{CD}^{+} \mathrm{T}$ cells to fight against microbes. If too late, microbes have already reached levels out of control. Triggering of the Hippo signaling pathway can certify the accurate regulation of differentiation of activated $\mathrm{CD} 8^{+} \mathrm{T}$ cells. After being activated by antigen and cytokines, the membrane expression of CD80 and CTLA-4 is upregulated and the combination of CD80 and CTLA- 4 in activated CD8 ${ }^{+}$T cells will then activate Mst1, leading to the phosphorylation and degradation of YAP through Lats $1 / 2$ $(58,63)$. Degradation of phosphorylated YAP contributes to the higher expression of Blimp-1, which mediates the terminal differentiation of $\mathrm{CD}^{+} \mathrm{T}$ cells (64). Moreover, CTLA-4-deficient $\mathrm{CD}^{+} \mathrm{T}$ cells do not downregulate YAP protein levels or upregulate Blimp-1 expression, demonstrating a key role for the CTLA-4 signaling axis in regulating canonical Hippo signaling and the induction of Blimp-1 in $\mathrm{CD}^{+} \mathrm{T}$ cells. It is also reported that, in primary $\mathrm{CD}^{+} \mathrm{T}$ cells, Mst1 deficiency leads to the impaired expression of FoxO3a, which induces the expression of the proapoptotic protein Bim. This dysregulation contributes to the excessive proliferation and delayed differentiation of primary $\mathrm{CD}^{+} \mathrm{T}$ cells in the absence of Mst1 (65). The probability of CD80/CTLA-4 combination is proportional to the cell density. Since the volume is fixed, the activation of Mst1 is proportional to the activated cell numbers, which makes it possible for Mst1 to control the timed terminal differentiation of $\mathrm{CD} 8^{+} \mathrm{T}$ cells. In summary, interaction between activated $\mathrm{CD}^{+} \mathrm{T}$ cells mediated by CD 80 and CTLA- 4 can induce the activation of Mst1, leading to the degradation of phosphorylated Yap and the upregulation of FoxO3a and Blimp-1 to promote their terminal differentiation.

Activated $M s t 1^{-1-} \mathrm{T}$ cells produce lower levels of the Th1related cytokines IL-2 and IFN- $\gamma$ and increased levels of the Th2 cytokine IL-4, indicating that $M s t 1^{-1-}$ naïve $\mathrm{CD}^{+} \mathrm{T}$ cells are inclined to differentiate toward Th2 cells. This biased differentiation may create an IL-4-rich environment which contributes to the activation, proliferation, and differentiation of plasmacytes, accounting for the hypergammaglobulinemia of $M s t 1^{-1-}$ mice. Indeed, $M s t 1^{-/-}$naïve T cells can promote the activation of B cells and plasma cell differentiation, resulting in a higher serum level of IgG, IgA and IgE (66). CD40-CD40L interactions on respective $\mathrm{B}$ cells and $\mathrm{T}$ cells provide a crucial second signal for $\mathrm{B}$ cell activation; however, $\mathrm{Mst}^{-1-} \mathrm{CD} 4^{+} \mathrm{T}$ cells do not express aberrant levels of CD40L.
Mst1 can also indirectly influence IL-17-producing Th17 cell differentiation. DC can modulate the differentiation of Th17 by producing IL-6, which is dependent upon Mst1 (67). Mechanistically, Mst1 deficiency in DC leads to increased LPSinduced p38 activation, which increases the production of IL-6. The IL-6-rich environment induces more IL-6R expression in the membrane of $\mathrm{CD}^{+} \mathrm{T}$ cells. Subsequently, IL-6R stimulation by IL-6 activates STAT3, a key transcription factor that promotes naive $\mathrm{CD}^{+} \mathrm{T}$ cell differentiation into Th17 cells (68). Taken together, these studies demonstrate that Mst1 can shape the adaptive immune response by regulating the differentiation of naïve $\mathrm{T}$ cells into specific subsets and Treg cell development and function.

\section{COMPARISON OF HIPPO SIGNALING BETWEEN OTHER TISSUES AND THE IMMUNE SYSTEM}

As proposed at the beginning of the article, canonical Hippo signaling regulates organ size by inhibiting tissue cell proliferation and promoting differentiation. Depletion of Mst1/2 in liver cells results in hepatomegaly and liver cancer, with an increased population of atypical ductal cells that bear characteristics of hepatic progenitors (69). At the cellular level, $M s t 1 / 2^{-1-}$ converts the cuboidal hepatocytes to atypical cells that behave like hepatic progenitors. The molecular mechanism underlying this process is that Mst1/2 deletion enhances the level of YAP through the canonical Hippo signal pathway. YAP translocates to the nucleus and interacts with TEAD1-4, which leads to the re-programming of adult hepatocytes and results in the de-differentiation of mature hepatocytes. Additionally, it is reported that the NOTCH pathway, known as a vital regulator of biliary cell fates during embryogenesis, is one of effectors of activated YAP. It is of significant interest to determine the other effectors of YAP in this Hippo signaling pathway as a means to limit liver cancer development and induce liver regeneration. Unlike the liver, the heart tissue does not undergo self-renewal when injured. However, cardiomyocytes deficient for Mst1/2 show an improved regenerative ability because YAP-dependent gene transcription linked to F-actin polymerization and cell cycle progression is increased (70). By comparison, $M s t 1 / 2^{-/-} \mathrm{T}$ cells have impaired F-actin polymerization, cell mobility, and cell proliferation with cell cycle progression being partially blocked at the G1/S transition.

Why does the Hippo signal pathway function differently between immune system and organs systems like the liver and heart? One explanation may lie in the different upstream receptor pathways that trigger Hippo signaling. In the canonical Hippo signaling pathway as shown in Figure 1, cell polarity, cell contact, extracellular mechanical signals, rearrangement of cytoskeleton, and GPCRs can regulate the activation of the Hippo pathway, allowing Hippo to regulate organ size (6, 44-46). Mst1/2 can be phosphorylated by the adjunction molecules, Mer and $\mathrm{Ex}$, in polar cells to promote cell differentiation and inhibit cell proliferation. GPCR signals induced by SIP inhibit Mst1/2 phosphorylation, which is reported to be related with the F-actin remodeling, leading to YAP activation. However, this situation is different in lymphocytes, where TCR or chemokine stimulation 
(including S1P) promotes Mst1 phosphorylation by Rap1 (or other unknown molecules) to positively regulate the rearrangement of actin and LFA- 1 clustering to induce T cell adhesion. As for the regulation of proliferation and apoptosis of lymphocytes, no uniform conclusion has been reached. Reports regarding how Mst1 is activated in lymphocytes are limited. Whether cell-cell interactions between lymphocytes or some other factors reflecting the expansion of lymphocytes can affect the activity of Mst1 is important for us to understand the role of Hippo signaling in regulation of lymphocyte proliferation and differentiation. In addition to potential differences in upstream regulators, Mst1/2 can regulate the activation of downstream targets in the immune system that are different than canonical Hippo targets activated in tissues, like liver. For instance, with the exception of CD80 and CTLA- 4 noted above, Lats $1 / 2$ and YAP activity are not regulated by Mst1 in lymphocytes.

Taken together, triggering of the Hippo pathway is very different between lymphocytes and traditional tissues, such as the liver and heart. The latter is regulated by F-actin mediated Hippo activation, while the former is Mst1-mediated actin polymerization. As for the regulation of proliferation and apoptosis in lymphocytes, more research is needed to address the precise role of Mst1 in this process. Therefore, the differences of Mst1 signal between the immune system and traditional tissues may due to its lack of understanding, or may be due to the differences between adherent cells in the environment.

\section{CONCLUDING REMARKS}

The conserved Hippo signaling pathway discovered in Drosophila plays essential roles in the generation of organs and development of cancer. Because of the special way it perceives signals, it can sense the mechanical cues outside the cell, including cell-cell interactions, which allows it to participate in the regulation of organ growth. Recent studies have also highlighted key roles for the mammalian Hippo kinases for the protection from aberrant immune reactions that can cause $\mathrm{T}$ cell-related inflammatory infiltration of multiple organs and auto-antibodies production. The function of Mst1 in the process of lymphocyte migration, development, activation, proliferation, and differentiation is gradually becoming more understood. However, many details in these processes are not clear. Except for RAPL/Rap1, are there other molecules regulating the activation of Mst1 in TCR and chemoattractant signaling pathways? In the immune system, can the cytoskeleton affect the activation of Mst1, as has been reported for the Hippo signaling pathway $(6,71-73)$ ? The molecular mechanisms of Mst1 in lymphocyte proliferation and apoptosis are still not fully understood, but are important to address because the canonical Hippo signal pathway is a key regulator of these processes in organ systems. Although Mst1/2 do not usually appear to signal via Lats $1 / 2$ or YAP in immune cells, is it possible that there are homologous molecules of Lats1/2, Mob1A/B, and $\mathrm{YAP} / \mathrm{TAZ}$ in immune system participating the survival signal of lymphocytes? Additionally, what others subsets of $\mathrm{T}$ cells are tightly associated with Mst1 and how does Mst1 function in these subsets? In summary, studies to date suggest primarily non-canonical roles for the Mst1 signaling pathway is different in immune system. Decoding the activators and effectors of Mst1 in the immune system will provide cues toward the treatment of some autoimmune disease and immunodeficiency disorders and improve the development of novel drug therapies.

\section{NOMENCLATURE}

\begin{tabular}{|c|c|}
\hline APC & T cell-antigen-presenting cell \\
\hline Akt & protein kinase $\mathrm{B}$ \\
\hline ADAP & adhesion and degranulation promoting adapter protein \\
\hline Blimp-1 & PR domain zinc finger protein 1 \\
\hline GPCR & G protein-coupled receptor \\
\hline $\mathrm{Bcl}-2$ & B-cell lymphoma 2 \\
\hline cTECS & cortex thymic epithelial cells \\
\hline CCL21 & chemokine (C-C motif) ligand 21 \\
\hline CTLA-4 & cytotoxic T-lymphocyte-associated protein 4 \\
\hline CD28 & cluster of differentiation 28 \\
\hline CD80 & cluster of differentiation 80 \\
\hline CD4 & cluster of differentiation 4 \\
\hline CD8 & cluster of differentiation 8 \\
\hline DP & double positive \\
\hline Dock8 & dedicator of cytokinesis 8 \\
\hline DENNC1C & differentially expressed in normal and neoplastic cell domain $1 \mathrm{C}$ \\
\hline DC & dendritic cell \\
\hline Erk & extracellular signal-regulated kinases \\
\hline Foxo1/3 & forkhead box protein $01 / 3$ \\
\hline FoxP3 & forkhead box P3 \\
\hline GEF & guanine nucleotide exchange factor \\
\hline GPCRs & G-protein-coupled receptors \\
\hline Hро & Hippo \\
\hline IkB & nuclear factor kappa-light-chain-enhancer of activated B cells \\
\hline IL2 & interleukin 2 \\
\hline IL-6 & interleukin 6 \\
\hline |L-17 & interleukin 17 \\
\hline IL-4 & interleukin 4 \\
\hline IFN-e & interferon gamma \\
\hline Lck & lymphocyte-specific protein tyrosine kinase \\
\hline ZAP-70 & zeta-chain-associated protein kinase 70 \\
\hline LPL & actin-bundling protein $\mathrm{L}$ plastin \\
\hline JNK & C-Jun N-terminal kinases \\
\hline LFA-1 & lymphocyte function-associated antigen 1 \\
\hline Mst1/2 & mammalian sterile 20-like kinase \\
\hline Mob1A/B & Mps one binder kinase activator-like 1 \\
\hline Mats & Mob as tumor suppressor \\
\hline MFI & mean fluorescence intensity \\
\hline mTECS & medullary thymic epithelial cells \\
\hline NDR1/2 & nuclear Dbf2-related 1/2, homolog of Lats \\
\hline Nrp1 & neuropilin-1 \\
\hline PPXY & Pro-Pro-Xaa-Tyr \\
\hline $\mathrm{pMHC}$ & peptide-majorhistocompatibility complex \\
\hline PD-1 & programmed cell death protein 1 \\
\hline p38 & mitogen-activated protein kinases \\
\hline PLC $\gamma 1$ & phosphoinositide phospholipase C \\
\hline Rac1 & Ras-related C3 botulinum toxin substrate 1 \\
\hline RhoA & Ras homolog gene family, member A \\
\hline Rab13 & a member of small Ras-related GTP binding protein family \\
\hline RAPL & $\begin{array}{l}\text { regulator for cell adhesion and polarization enriched in lymphoid } \\
\text { tissue (also known as RASSF5) }\end{array}$ \\
\hline SP & single positive \\
\hline Sav & salvador \\
\hline Sd & scalloped \\
\hline Salv & salvador homolog 1 \\
\hline Lats $1 / 2$ & large tumor suppressor homolog $1 / 2$ \\
\hline STE20 & sterile 20 \\
\hline
\end{tabular}

(Continued) 


$\begin{array}{ll}\text { SARAH } & \text { Sav/Rassf/Hpo } \\ \text { S1P } & \text { sphingosine-1phosphate } \\ \text { SKAP1/SKAP5 } & \text { Src kinase-associated phosphoprotein 1/55 } \\ \text { SLP-76 } & \text { lymphocyte cytosolic protein 2 } \\ \text { TEAD1-4 } & \text { TEA domain family member 1 to 4 } \\ \text { Treg } & \text { regulatory T cells } \\ \text { TCR } & \text { T cell receptor } \\ \text { Th1 } & \text { Thelper cells 1 } \\ \text { Th2 } & \text { Thelper cells 2 } \\ \text { Th17 } & \text { Thelper cells 17 } \\ \text { TGF-beta } & \text { transforming growth factor beta 1 } \\ \text { VASP } & \text { vasodilator-stimulated phosphorylation } \\ \text { Wts } & \text { Warts } \\ \text { Yki } & \text { Yorkie } \\ \text { YAP/TAZ } & \text { Yes-associated protein/transcriptional coactivator with PDZ- } \\ & \text { binding motif } \\ \text { 14-3-3 } & \text { a family of conserved regulatory molecules that are expressed } \\ & \text { in all eukaryotic cells }\end{array}$

\section{REFERENCES}

1. Justice RW, Zilian O, Woods DF, Noll M, Bryant PJ. The Drosophila tumor suppressor gene warts encodes a homolog of human myotonic dystrophy kinase and is required for the control of cell shape and proliferation. Genes Dev (1995) 9:534-46. doi:10.1101/gad.9.5.534

2. Boedigheimer M, Laughon A. Expanded: a gene involved in the control of cell proliferation in imaginal discs. Development (1993) 118:1291-301.

3. Watson KL, Justice RW, Bryant PJ. Drosophila in cancer research: the first fifty tumor suppressor genes. J Cell Sci Supplement (1994) 18:19-33. doi:10.1242/ jcs.1994.Supplement_18.4

4. Lai ZC, Wei X, Shimizu T, Ramos E, Rohrbaugh M, Nikolaidis N, et al. Control of cell proliferation and apoptosis by mob as tumor suppressor, mats. Cell (2005) 120:675-85. doi:10.1016/j.cell.2004.12.036

5. Dong J, Feldmann G, Huang J, Wu S, Zhang N, Comerford SA, et al. Elucidation of a universal size-control mechanism in drosophila and mammals. Cell (2007) 130:1120-33. doi:10.1016/j.cell.2007.07.019

6. Zhao B, Li L, Wang L, Wang CY, Yu J, Guan KL. Cell detachment activates the Hippo pathway via cytoskeleton reorganization to induce anoikis. Genes Dev (2012) 26:54-68. doi:10.1101/gad.173435.111

7. Scheel H, Hofmann K. A novel interaction motif, SARAH, connects three classes of tumor suppressor. Curr Biol (2003) 13:R899-900. doi:10.1016/j.cub.2003. 11.007

8. Pantalacci S, Tapon N, Leopold P. The salvador partner hippo promotes apoptosis and cell-cycle exit in drosophila. Nat Cell Biol (2003) 5:921-7. doi:10.1038/ncb1051

9. Hwang E, Cheong HK, Ul Mushtaq A, Kim HY, Yeo KJ, Kim E, et al. Structural basis of the heterodimerization of the MST and RASSF SARAH domains in the hippo signalling pathway. Acta Crystallogr D Biol Crystallogr (2014) 70:1944-53. doi:10.1107/S139900471400947X

10. Praskova M, Xia F, Avruch J. MOBKL1A/MOBKL1B phosphorylation by MST1 and MST2 inhibits cell proliferation. Curr Biol (2008) 18:311-21. doi:10.1016/j.cub.2008.02.006

11. Huang J, Wu S, Barrera J, Matthews K, Pan D. The hippo signaling pathway coordinately regulates cell proliferation and apoptosis by inactivating yorkie, the drosophila homolog of YAP. Cell (2005) 122:421-34. doi:10.1016/j. cell.2005.06.007

12. Edgar BA. From cell structure to transcription: hippo forges a new path. Cell (2006) 124:267-73. doi:10.1016/j.cell.2006.01.005

13. Shi Z, Jiao S, Zhou Z. Structural dissection of hippo signaling. Chin J Biochem Biophys (2015) 47:29-38. doi:10.1093/abbs/gmu107

14. Avruch J, Zhou D, Fitamant J, Bardeesy N, Mou F, Barrufet LR. Protein kinases of the Hippo pathway: regulation and substrates. Semin Cell Dev Biol (2012) 23:770-84. doi:10.1016/j.semcdb.2012.07.002

15. Zhao B, Wei X, Li W, Udan RS, Yang Q, Kim J, et al. Inactivation of YAP oncoprotein by the Hippo pathway is involved in cell contact inhibition and tissue growth control. Genes Dev (2007) 21:2747-61. doi:10.1101/gad.1602907

\section{AUTHOR CONTRIBUTIONS}

CL organized the article. JC wrote the draft. YJ, DK, LY, ZY, ZP, $\mathrm{XL}, \mathrm{YW}$, and QG revised the draft. JL drew the figures. RM and YZ edited the language and figures.

\section{ACKNOWLEDGMENTS}

We gratefully thank Yanan Gao for giving many useful advices and Nicole M. Chapman for editing the manuscript.

\section{FUNDING}

This work was supported by Natural Science Foundation of China (81722002 and 31500709).

16. Zhao B, Ye X, Yu J, Li L, Li W, Li S, et al. TEAD mediates YAP-dependent gene induction and growth control. Genes Dev (2008) 22:1962-71. doi:10.1101/ gad.1664408

17. Zhou Y, Huang T, Cheng AS, Yu J, Kang W, To KF. The TEAD Family and Its Oncogenic Role in Promoting Tumorigenesis. Int J Mol Sci (2016) 17:E138. doi:10.3390/ijms17010138

18. Yimlamai D, Fowl BH, Camargo FD. Emerging evidence on the role of the Hippo/YAP pathway in liver physiology and cancer. J Hepatol (2015) 63:1491-501. doi:10.1016/j.jhep.2015.07.008

19. Ma Y, Yang Y, Wang F, Wei Q, Qin H. Hippo-YAP signaling pathway: a new paradigm for cancer therapy. Int J Cancer (2015) 137:2275-86. doi:10.1002/ ijc. 29073

20. You B, Yang YL, Xu Z, Dai Y, Liu S, Mao JH, et al. Inhibition of ERK1/2 down-regulates the Hippo/YAP signaling pathway in human NSCLC cells. Oncotarget (2015) 6:4357-68. doi:10.18632/oncotarget.2974

21. Witt CM, Raychaudhuri S, Schaefer B, Chakraborty AK, Robey EA. Directed migration of positively selected thymocytes visualized in real time. PLoS Biol (2005) 3:e160. doi:10.1371/journal.pbio.0030160

22. Takahama Y. Journey through the thymus: stromal guides for T-cell development and selection. Nat Rev Immunol (2006) 6:127-35. doi:10.1038/nri1781

23. Sakaguchi S, Yamaguchi T, Nomura T, Ono M. Regulatory T cells and immune tolerance. Cell (2008) 133:775-87. doi:10.1016/j.cell.2008.05.009

24. Dong Y, Du X, Ye J, Han M, Xu T, Zhuang Y, et al. A cell-intrinsic role for Mst1 in regulating thymocyte egress. J Immunol (2009) 183:3865-72. doi:10.4049/ jimmunol.0900678

25. Mou F, Praskova M, Xia F, Van Buren D, Hock H, Avruch J, et al. The Mstl and Mst2 kinases control activation of rho family GTPases and thymic egress of mature thymocytes. J Exp Med (2012) 209:741-59. doi:10.1084/jem.20111692

26. Ueda Y, Katagiri K, Tomiyama T, Yasuda K, Habiro K, Katakai T, et al. Mst1 regulates integrin-dependent thymocyte trafficking and antigen recognition in the thymus. Nat Commun (2012) 3:1098. doi:10.1038/ncomms2105

27. Zhou D, Medoff BD, Chen L, Li L, Zhang XF, Praskova M, et al. The Nore1B/ Mst1 complex restrains antigen receptor-induced proliferation of naive T cells. Proc Nat Acad Sci U S A (2008) 105:20321-6. doi:10.1073/pnas.0810773105

28. Tang F, Gill J, Ficht X, Barthlott T, Cornils H, Schmitz-Rohmer D, et al. The kinases NDR1/2 act downstream of the hippo homolog MST1 to mediate both egress of thymocytes from the thymus and lymphocyte motility. Sci Signal (2015) 8:ra100. doi:10.1126/scisignal.aab2425

29. Krammer PH, Arnold R, Lavrik IN. Life and death in peripheral T cells. Nat Rev Immunol (2007) 7:532-42. doi:10.1038/nri2115

30. Tomiyama T, Ueda Y, Katakai T, Kondo N, Okazaki K, Kinashi T. Antigenspecific suppression and immunological synapse formation by regulatory T cells require the Mst1 kinase. PLoS One (2013) 8:e73874. doi:10.1371/ journal.pone.0073874

31. Du X, Shi H, Li J, Dong Y, Liang J, Ye J, et al. Mst1/Mst2 regulate development and function of regulatory $\mathrm{T}$ cells through modulation of Foxo1/Foxo3 
stability in autoimmune disease. J Immunol (2014) 192:1525-35. doi:10.4049/ jimmunol.1301060

32. Dustin ML, Bivona TG, Philips MR. Membranes as messengers in T cell adhesion signaling. Nat Immunol (2004) 5:363-72. doi:10.1038/ ni1057

33. Katagiri K, Imamura M, Kinashi T. Spatiotemporal regulation of the kinase Mst1 by binding protein RAPL is critical for lymphocyte polarity and adhesion. Nat Immunol (2006) 7:919-28. doi:10.1038/ni1374

34. Wang H, Wei B, Bismuth G, Rudd CE. SLP-76-ADAP adaptor module regulates LFA-1 mediated costimulation and T cell motility. Proc Nat Acad Sci U S A (2009) 106:12436-41. doi:10.1073/pnas.0900510106

35. Wang H, McCann FE, Gordan JD, Wu X, Raab M, Malik TH, et al. ADAPSLP-76 binding differentially regulates supramolecular activation cluster (SMAC) formation relative to T cell-APC conjugation. JExp Med (2004) 200:1063-74. doi:10.1084/jem.20040780

36. Wang H, Liu H, Lu Y, Lovatt M, Wei B, Rudd CE. Functional defects of SKAP-55-deficient T cells identify a regulatory role for the adaptor in LFA-1 adhesion. Mol Cell Biol (2007) 27:6863-75. doi:10.1128/ MCB.00556-07

37. Raab M, Wang H, Lu Y, Smith X, Wu Z, Strebhardt K, et al. T cell receptor "inside-out" pathway via signaling module SKAP1-RapL regulates $\mathrm{T}$ cell motility and interactions in lymph nodes. Immunity (2010) 32:541-56. doi:10.1016/j.immuni.2010.03.007

38. Kliche S, Worbs T, Wang X, Degen J, Patzak I, Meineke B, et al. CCR7mediated LFA-1 functions in T cells are regulated by 2 independent ADAP/SKAP55 modules. Blood (2012) 119:777-85. doi:10.1182/ blood-2011-06-362269

39. Raab M, Smith X, Matthess Y, Strebhardt K, Rudd CE. SKAP1 protein PH domain determines RapL membrane localization and Rap1 protein complex formation for T cell receptor (TCR) activation of LFA-1. J Biol Chem (2011) 286:29663-70. doi:10.1074/jbc.M111.222661

40. Menasche G, Kliche S, Chen EJ, Stradal TE, Schraven B, Koretzky G. RIAM links the ADAP/SKAP-55 signaling module to Rap1, facilitating T-cellreceptor-mediated integrin activation. Mol Cell Biol (2007) 27:4070-81. doi:10.1128/MCB.02011-06

41. Kondo N, Ueda Y, Kita T, Ozawa M, Tomiyama T, Yasuda K, et al. NDR1Dependent regulation of kindlin-3 controls high-affinity LFA-1 binding and immune synapse organization. Mol Cell Biol (2017) 37:e424-416. doi:10.1128/ MCB.00424-16

42. Katagiri K, Katakai T, Ebisuno Y, Ueda Y, Okada T, Kinashi T. Mst1 controls lymphocyte trafficking and interstitial motility within lymph nodes. EMBO J (2009) 28:1319-31. doi:10.1038/emboj.2009.82

43. Kanda I, Nishimura N, Nakatsuji H, Yamamura R, Nakanishi H, Sasaki T. Involvement of Rab13 and JRAB/MICAL-L2 in epithelial cell scattering. Oncogene (2008) 27:1687-95. doi:10.1038/sj.onc.1210812

44. Katagiri K, Maeda A, Shimonaka M, Kinashi T. RAPL, a Rap1-binding molecule that mediates Rap1-induced adhesion through spatial regulation of LFA-1. Nat Immunol (2003) 4:741-8. doi:10.1038/ni950

45. Nishikimi A, Ishihara S, Ozawa M, Etoh K, Fukuda M, Kinashi T, et al. Rab13 acts downstream of the kinase Mst1 to deliver the integrin LFA-1 to the cell surface for lymphocyte trafficking. Sci Signal (2014) 7:ra72. doi:10.1126/ scisignal.2005199

46. Yoshimura S, Gerondopoulos A, Linford A, Rigden DJ, Barr FA. Family-wide characterization of the DENN domain Rab GDP-GTP exchange factors. J Cell Biol (2010) 191:367-81. doi:10.1083/jcb.201008051

47. Doppler HR, Bastea LI, Lewis-Tuffin LJ, Anastasiadis PZ, Storz P. Protein kinase D1-mediated phosphorylations regulate vasodilator-stimulated phosphoprotein (VASP) localization and cell migration. J Biol Chem (2013) 288:24382-93. doi:10.1074/jbc.M113.474676

48. Choudhuri K, Llodra J, Roth EW, Tsai J, Gordo S, Wucherpfennig KW, et al. Polarized release of T-cell-receptor-enriched microvesicles at the immunological synapse. Nature (2014) 507:118-23. doi:10.1038/nature12951

49. Finetti F, Paccani SR, Riparbelli MG, Giacomello E, Perinetti G, Pazour GJ, et al. Intraflagellar transport is required for polarized recycling of the TCR/ CD3 complex to the immune synapse. Nat Cell Biol (2009) 11:1332-9. doi: $10.1038 /$ ncb 1977

50. Ridley AJ, Schwartz MA, Burridge K, Firtel RA, Ginsberg MH, Borisy G, et al. Cell migration: integrating signals from front to back. Science (2003) 302:1704-9. doi:10.1126/science.1092053
51. Xu X, Wang X, Todd EM, Jaeger ER, Vella JL, Mooren OL, et al. Mst1 kinase regulates the actin-bundling protein L-plastin to promote $\mathrm{T}$ cell migration. J Immunol (2016) 197:1683-91. doi:10.4049/jimmunol.1600874

52. Xu X, Jaeger ER, Wang X, Lagler-Ferrez E, Batalov S, Mathis NL, et al. Mst1 directs Myosin IIa partitioning of low and higher affinity integrins during T cell migration. PLoS One (2014) 9:e105561. doi:10.1371/journal.pone.0105561

53. Gomez TS, Billadeau DD. T cell activation and the cytoskeleton: you can't have one without the other. Adv Immunol (2008) 97:1-64. doi:10.1016/ S0065-2776(08)00001-1

54. Meller N, Merlot S, Guda C. CZH proteins: a new family of Rho-GEFs. J Cell Sci (2005) 118:4937-46. doi:10.1242/jcs.02671

55. Du X, Yu A, Tao W. The non-canonical Hippo/Mst pathway in lymphocyte development and functions. Chin J Biochem Biophys (2015) 47:60-4. doi:10.1093/abbs/gmu112

56. Salojin KV, Hamman BD, Chang WC, Jhaver KG, Al-Shami A, Crisostomo J, et al. Genetic deletion of Mst1 alters T cell function and protects against autoimmunity. PLoS One (2014) 9:e98151. doi:10.1371/journal.pone.0098151

57. Choi J, Oh S, Lee D, Oh HJ, Park JY, Lee SB, et al. Mst1-FoxO signaling protects Naive T lymphocytes from cellular oxidative stress in mice. PLoS One (2009) 4:e8011. doi:10.1371/journal.pone.0008011

58. Thaventhiran JE, Hoffmann A, Magiera L, de la Roche M, Lingel H, BrunnerWeinzierl M, et al. Activation of the Hippo pathway by CTLA-4 regulates the expression of Blimp-1 in the CD8+ T cell. Proc Natl Acad Sci U S A (2012) 109:E2223-9. doi:10.1073/pnas.1209115109

59. Fontenot JD, Gavin MA, Rudensky AY. Foxp3 programs the development and function of CD4+CD25+ regulatory T cells. Nat Immunol (2003) 4:330-6. doi:10.1038/ni904

60. vanLoosdregtJ, Vercoulen Y,GuichelaarT, GentYY,BeekmanJM,vanBeekumO, et al. Regulation of treg functionality by acetylation-mediated Foxp3 protein stabilization. Blood (2010) 115:965-74. doi:10.1182/blood-2009-02-207118

61. Li J, Du X, Shi H, Deng K, Chi H, Tao W. Mammalian Sterile 20-like Kinase 1 (Mst1) enhances the stability of Forkhead Box P3 (Foxp3) and the function of regulatory T Cells by modulating Foxp3 acetylation. J Biol Chem (2015) 290:30762-70. doi:10.1074/jbc.M115.668442

62. Lehtinen MK, Yuan Z, Boag PR, Yang Y, Villen J, Becker EB, et al. A conserved MST-FOXO signaling pathway mediates oxidative-stress responses and extends life span. Cell (2006) 125:987-1001. doi:10.1016/j.cell.2006.03.046

63. Pentcheva-Hoang T, Egen JG, Wojnoonski K, Allison JP. B7-1 and B7-2 selectively recruit CTLA-4 and CD28 to the immunological synapse. Immunity (2004) 21:401-13. doi:10.1016/j.immuni.2004.06.017

64. Rutishauser RL, Martins GA, Kalachikov S, Chandele A, Parish IA, Meffre E, et al. Transcriptional repressor Blimp-1 promotes CD8(+) T cell terminal differentiation and represses the acquisition of central memory $\mathrm{T}$ cell properties. Immunity (2009) 31:296-308. doi:10.1016/j.immuni.2009.05.014

65. Yasuda K, Ueda Y, Ozawa M, Matsuda T, Kinashi T. Enhanced cytotoxic T-cell function and inhibition of tumor progression by Mst1 deficiency. FEBS Lett (2016) 590:68-75. doi:10.1002/1873-3468.12045

66. Park E, Kim MS, Song JH, Roh KH, Lee R, Kim TS. MST1 deficiency promotes $\mathrm{B}$ cell responses by $\mathrm{CD} 4+\mathrm{T}$ cell-derived IL-4, resulting in hypergammaglobulinemia. Biochem Biophys Res Commun (2017) 489:56-62. doi:10.1016/j. bbrc.2017.05.094

67. Jones GW, McLoughlin RM, Hammond VJ, Parker CR, Williams JD, Malhotra R, et al. Loss of CD4+ T cell IL-6R expression during inflammation underlines a role for IL- 6 trans signaling in the local maintenance of Th17 cells. J Immunol (2010) 184:2130-9. doi:10.4049/jimmunol.0901528

68. Li C, Bi Y, Li Y, Yang H, Yu Q, Wang J, et al. Dendritic cell MST1 inhibits Th17 differentiation. Nat Commun (2017) 8:14275. doi:10.1038/ncomms14275

69. Yimlamai D, Christodoulou C, Galli GG, Yanger K, Pepe-Mooney B, Gurung B, et al. Hippo pathway activity influences liver cell fate. Cell (2014) 157:1324-38. doi:10.1016/j.cell.2014.03.060

70. Morikawa Y, Zhang M, Heallen T, Leach J, Tao G, Xiao Y, et al. Actin cytoskeletal remodeling with protrusion formation is essential for heart regeneration in hippo-deficient mice. Sci Signal (2015) 8:ra41. doi:10.1126/scisignal.2005781

71. Yu FX, Zhao B, Panupinthu N, Jewell JL, Lian I, Wang LH, et al. Regulation of the Hippo-YAP pathway by G-protein-coupled receptor signaling. Cell (2012) 150:780-91. doi:10.1016/j.cell.2012.06.037

72. Wada K, Itoga K, Okano T, Yonemura S, Sasaki H. Hippo pathway regulation by cell morphology and stress fibers. Development (2011) 138:3907-14. doi:10.1242/dev.070987 
73. Mo JS, Yu FX, Gong R, Brown JH, Guan KL. Regulation of the Hippo-YAP pathway by protease-activated receptors (PARs). Genes Dev (2012) 26:2138-43. doi:10.1101/gad.197582.112

Conflict of Interest Statement: We declare that the research was conducted in the absence of any commercial or financial relationships that could be construed as a potential conflict of interest.
Copyright $\odot 2018$ Cheng, Jing, Kang, Yang, Li, Yu, Peng, Li, Wei, Gong, Miron, Zhang and Liu. This is an open-access article distributed under the terms of the Creative Commons Attribution License (CC BY). The use, distribution or reproduction in other forums is permitted, provided the original author(s) and the copyright owner are credited and that the original publication in this journal is cited, in accordance with accepted academic practice. No use, distribution or reproduction is permitted which does not comply with these terms. 